\title{
Reinforcement schedule effects in rats trained to discriminate 3,4-methylenedioxymethamphetamine (MDMA) or cocaine
}

\author{
Daniel Kueh • Lisa E. Baker
}

Received: 10 December 2005 / Accepted: 21 July 2006 / Published online: 23 September 2006

(C) Springer-Verlag 2006

\begin{abstract}
Rationale Relatively few studies have compared the discriminative stimulus effects of 3,4-methylenedioxymethamphetamine (MDMA) and cocaine, and findings from different laboratories are somewhat inconsistent. One possible reason for discrepant results may be the use of different reinforcement schedules during discrimination training.

Objective The present study compared fixed ratio (FR) 20 and variable interval (VI) 15-s reinforcement schedules to determine their influence on discrimination acquisition, response rates, frequency of reinforcements, and stimulus generalization in rats trained to discriminate cocaine or MDMA.

Materials and methods Thirty-two male Sprague-Dawley rats were trained to discriminate cocaine $(10 \mathrm{mg} / \mathrm{kg} ; n=16)$ or MDMA $(1.5 \mathrm{mg} / \mathrm{kg} ; n=16)$ from saline under either a FR 20 or a VI 15-s schedule of food reinforcement. Stimulus generalization tests were conducted with a range of doses of cocaine, MDMA, d-amphetamine, and lysergic acid diethylamide in all four training groups.

Results The FR 20 schedule facilitated more rapid discrimination acquisition compared to the VI 15-s schedule and established differential response rates and frequency of reinforcement under drug and vehicle conditions. However,
\end{abstract}

L. E. Baker $(\bowtie)$

Department of Psychology,

Western Michigan University,

Kalamazoo, MI 49008, USA

e-mail: lisa.baker@wmich.edu

D. Kueh

Department of Biological Sciences,

Western Michigan University,

Kalamazoo, MI 49008, USA reinforcement schedule had little influence on stimulus generalization between MDMA and cocaine. Cocaine produced partial substitution for MDMA in both training groups (FR 20, 51\%; VI 15-s, 58\%). Likewise, MDMA produced only partial substitution for cocaine in both training groups (FR 20, 40\%; VI 15-s, 72\%).

Conclusions The present findings suggest that the number of sessions required to establish discriminative stimulus control varies with different reinforcement schedules. Nevertheless, training schedules alone do not appear to have significant effects on stimulus generalization between MDMA and cocaine.

Keywords MDMA - Cocaine - Amphetamine - LSD . Drug discrimination · Reinforcement schedules · Rats . Fixed ratio $\cdot$ Variable interval $\cdot$ Methodology

\section{Introduction}

Drug discrimination is a well-established neurobehavioral assay frequently employed to classify psychoactive drugs, to compare novel and existing drugs, and to investigate neurochemical actions that mediate the discriminative stimulus effects of drugs. This popular assay has proven useful given that the discriminative stimulus effects of drugs in animals parallel the subjective drug effects in humans (Brauer et al. 1997; Goudie and Leathley 1993). Moreover, it has been shown to be a stable, specific, and sensitive measure of dose-related drug effects (Glennon et al. 1983).

Drug discrimination methodology varies widely among different laboratories. Some common variables include differences among the test subjects (e.g., species, strain, sex), differences in training procedures (e.g., type of 
reinforcer, schedule of reinforcement, number of alternative responses), differences in drug delivery (e.g., route of administration, training dose), and differences in test procedures (e.g., discrete vs cumulative dosing procedures, reinforced responding vs extinction, test duration). Although not all of these methodological variables have been examined extensively, there is evidence regarding the importance of some of these methodological variables. For example, White and Appel (1982) trained three groups of rats to discriminate three different doses of lysergic acid diethylamide (LSD; 0.02, 0.08, and $0.32 \mathrm{mg} / \mathrm{kg}$ ). These authors reported that the LSD training dose influenced results in subsequent dose-response tests, and the steepness of the slope in LSD dose-response curves was dependent upon the training dose. Stolerman and D'Mello (1981) reported similar results with different training doses of $(+)$ amphetamine $(0.4,1.0$, and $1.6 \mathrm{mg} / \mathrm{kg})$.

Other investigators have also examined the effects of strain differences in drug discrimination studies (e.g., Meehan et al. 1995; Schechter 1997, 1998). For example, Meehan et al. (1995) trained selectively bred high- and lowalcohol drinking (HAD/LAD) rats to discriminate ethanol $(600 \mathrm{mg} / \mathrm{kg})$ from vehicle. When exposed to different doses of MDMA $(0.5,1.0,1.5$, and $3.0 \mathrm{mg} / \mathrm{kg})$ during substitution tests, they found HAD animals were significantly more sensitive than LAD animals to the effects of MDMA. The influence of sex differences has also been examined in several drug discrimination investigations (e.g., Craft and Stratmann 1996; Craft et al. 1996, 1998; Anderson and van Haaren 1999, 2000). Studies of cocaine discrimination in rats have revealed little or no evidence for sex differences in cocaine generalization gradients or effective dose 50 $\left(\mathrm{ED}_{50}\right.$; Anderson and van Haaren 2000; Craft and Stratmann 1996). Initial reports of sex differences in the acquisition of morphine discrimination in rats (Craft et al. 1996) were later explained as probably due to a bias in reinforcement frequency that occurred in males, but not in females under an fixed ratio (FR) 10 schedule (Craft et al. 1998).

The schedule of reinforcement used during discrimination training is an especially crucial methodological variable. The reinforcement schedules most commonly used in drug discrimination research are FR schedules and variable interval (VI) schedules, and, to a lesser extent, fixed interval (FI) schedules. A survey by Stolerman (1989b) of 606 drug discrimination studies published between 1951 and 1986 showed that 396 studies used FR schedules of reinforcement and 115 studies used VI schedules of reinforcement. In the FR schedule, a fixed number of responses by a subject on the condition-appropriate lever are reinforced. A common variation of the FR schedule includes a resetting component that punishes lever switching behavior, whereby a fixed consecutive number of responses on one lever is required for reinforcement (e.g., Baker et al. 2004; Stolerman 1989b). The VI schedule, however, is dependent on the first response made after the passage of a variable interval of time. FR and VI schedules produce distinctly different patterns and rates of responding. Furthermore, FR schedules engender a greater frequency of reinforcement and a shorter delay between response and reinforcement delivery compared to VI schedules.

Several investigators have used a variety of reinforcement schedules and a limited number of drugs including barbiturates, phencyclidine, nicotine, and opiates to study the effects of reinforcement schedules on discriminative stimulus control by psychoactive drugs in rats (Overton 1979; DeVry et al. 1984; Stolerman 1989a,b; Snodgrass and McMillan 1991; Craft et al. 1998) or in pigeons (McMillan and Wenger 1984; McMillan et al. 2001). The main conclusion evident from these studies is that reinforcement schedules do influence stimulus control by drugs and that FR schedules tend to assert greater stimulus control compared to either VI or FI schedules. Reinforcement schedule-dependent effects on discriminative stimulus control may be attributed to differences in reinforcement frequency between drug and vehicle conditions. Indeed, previous research has documented reinforcement variables as determinants of drug generalization (Colpaert and Janssen 1981; Koek and Slangen et al. 1982) and the speed of drug discrimination acquisition (DeVry et al. 1984).

Although a number of studies have examined the influence of reinforcement schedules on drug discrimination, none thus far have examined the influence of reinforcement schedule on the development of stimulus control by MDMA or cocaine. Moreover, surprisingly few studies have compared the discriminative stimulus effects of these two commonly abused substances.

A few studies have compared cocaine to the optical isomers of MDMA. Broadbent et al. (1989) trained three different groups of rats to discriminate one of three doses of cocaine $(3.5,10,20 \mathrm{mg} / \mathrm{kg})$ from saline and assessed each MDMA isomer for stimulus generalization to each training dose of cocaine. Neither isomer substituted fully for the 3.5 or the $10 \mathrm{mg} / \mathrm{kg}$ cocaine training dose, and only (-)MDMA produced substitution for the $20 \mathrm{mg} / \mathrm{kg}$ cocaine dose, although only three of the five animals tested responded at this dose. Baker et al. (1995) later reported that cocaine produced only partial substitution (between 40 and $60 \%$ drug-appropriate responding) in rats trained to discriminate either $1.25 \mathrm{mg} / \mathrm{kg}(+)-\mathrm{MDMA}$ or $3.5 \mathrm{mg} / \mathrm{kg}$ $(-)$ MDMA from saline. More recently, Bondavera et al. (2005) reported that cocaine produced complete stimulus generalization in rats trained to discriminate a lower dose of (-)-MDMA $(1.75 \mathrm{mg} / \mathrm{kg})$ and also found that cocaine did not substitute for $0.75 \mathrm{mg} / \mathrm{kg}(+)$-MDMA. It is also noteworthy that Baker et al. (1995) used an FR 20 
schedule, while Bondavera et al. (2005) used a VI 15-s schedule of reinforcement during discrimination training.

To our knowledge, only two published drug discrimination studies have compared cocaine to $( \pm$ )-MDMA (hereafter referred to simply as MDMA). Khorana et al. (2004) compared the discriminative stimulus effects of cocaine and MDMA in Sprague-Dawley rats. Under a VI 15-s schedule of reinforcement, cocaine substituted for MDMA in rats trained to discriminate MDMA from saline, but MDMA failed to substitute in rats trained to discriminate cocaine from saline. The authors concluded that stimulus generalization between MDMA and cocaine is asymmetrical. These results are inconsistent with those of an earlier study by Schechter (1998). Using an FR 10 schedule of reinforcement, Schechter reported that cocaine did not substitute for MDMA in fawn-hooded rats trained to discriminate MDMA from saline. Khorana et al. (2004) explained this apparent inconsistency by pointing to the different methodological approaches and different strains of animals used in both studies. Although the difference in animal strain was a likely cause for the discrepant findings, it is also possible that other methodological variables such as the schedule of reinforcement may have contributed to the discrepant findings of these two studies.

The purpose of the present investigation was to compare two reinforcement schedules, FR 20 and VI 15-s, on the establishment of discriminative stimulus control by MDMA or cocaine and to examine the influence of reinforcement schedule on stimulus generalization between these compounds and to two other drugs, d-amphetamine and LSD.

\section{Materials and methods}

\section{Subjects}

Thirty-two male Sprague-Dawley rats (Charles River Laboratories, Portage, MI), 50- to 60-days-old at the beginning of the study were used. Animals were housed individually in polycarbonate cages lined with corncob bedding. They were acclimated to an animal colony maintained on a 12-h light/ 12-h dark cycle at a constant temperature $\left(20 \pm 2^{\circ} \mathrm{C}\right)$ and humidity $(50 \pm 5 \%)$ for 1 week before beginning the study. Water was available in the home cages ad libitum. After the 1-week acclimation period, food was restricted to reduce weights to approximately $80-85 \%$ of free-feeding weights. Subjects were maintained according to the general principles of animal husbandry as stated by the US Department of Health, Education, and Welfare (National Research Council 1996), and the research protocol was approved by the Western Michigan University Institutional Animal Care and Use Committee.
Apparatus

Training and testing sessions were conducted in eight operant conditioning chambers (MED Associates, Georgia, VT) measuring $28 \mathrm{~cm}$ long by $21 \mathrm{~cm}$ wide by $21 \mathrm{~cm}$ high. Each chamber contained two retractable levers, a food receptacle, a $28-\mathrm{V}$ white light to illuminate the chamber, and a fan to mask noise and provide ventilation. Reinforcers consisted of $45 \mathrm{mg}$ food pellets (BioServ \#F0021, Frenchtown, NJ). Programming and data event recordings were conducted with Med-PC software (version IV) installed on an IBM-compatible computer.

\section{Drugs}

$( \pm)$-3,4-methylenedioxymethamphetamine-hydrochloride, $(-)$-cocaine-hydrochloride, $(+)$-lysergic acid diethylamide and $(+)$-amphetamine sulfate were generously provided by the National Institute on Drug Abuse (Rockville, MD). All drugs were dissolved in $0.9 \%$ bacteriostatic sodium chloride and they were administrated by intraperitoneal (IP) injection using $1 \mathrm{ml}$ insulin syringes (Sherwood Medical, St. Louis, MO) 15 min before each training or test session. Drug doses were calculated based on the weight of the salt.

\section{Discrimination training and reinforcement schedules}

Subjects were divided into four training groups through random assignment. The COC-FR group was trained under an FR 20 schedule of reinforcement with cocaine $(10 \mathrm{mg} / \mathrm{kg})$ as the training drug. The COC-VI group was trained under a VI $15-\mathrm{s}$ schedule of reinforcement with cocaine $(10 \mathrm{mg} / \mathrm{kg})$ as the training drug. The MDMA-FR group was trained under an FR 20 schedule of reinforcement with MDMA $(1.5 \mathrm{mg} / \mathrm{kg})$ as the training drug. The MDMA-VI group was trained under a VI 15-s schedule of reinforcement with MDMA $(1.5 \mathrm{mg} / \mathrm{kg})$ as the training drug.

Before commencing drug discrimination training, all subjects were first exposed to a single 1-h session under a fixed time 60-s schedule of food delivery without the levers present. This procedure paired the sound of pellet drop with food availability, which was not contingent on any response. After this procedure, eight lever-press training sessions were conducted whereby all subjects were exposed to errorless training conditions (e.g., Baker et al. 2004). Under these conditions, only one of two levers was present, depending on whether drug or vehicle was administered. Subjects received 4 days of errorless training under the drug condition and 4 days of errorless training under the vehicle condition before discrimination training began. For half the animals in each training group, the right lever was designated as drug-appropriate and the left lever was 
designated as vehicle-appropriate. Lever assignments were reversed for the remaining animals in each group.

After the completion of errorless training, discrimination training for all subjects began on an FR 1 schedule with both levers present. Isopropyl alcohol was used to wipe both levers between training sessions to control for possible olfactory cues (Extance and Goudie 1981). Once subjects were reliably responding under both conditions, subsequent training was conducted under an FR 20 schedule for the COC-FR and MDMA-FR groups and under a VI 15-s schedule for the COC-VI and MDMA-VI groups. All subjects were trained 6 days a week during 15-min training sessions and in the order of VVDDVD, VVDVDD, DDVVDV, or DDVDVV, whereby " $D$ " is the drug session and " $\mathrm{V}$ " is the vehicle session.

Subjects trained under the FR 20 schedule were first exposed to an initial value of FR 1, which incremented progressively to a resetting FR 20 schedule. Depending on each subject's performance, the starting FR value per session was automatically incremented by $n$ after every fifth reinforcement, where $n$ could be programmed to any number from 1 to 19. The starting FR and increment values were the same for all subjects in the FR groups at the beginning of each session, and the FR value was systematically increased across sessions until all subjects were responding reliably under an FR 20 schedule under both drug and vehicle conditions.

For the other two groups of subjects, a VI 15-s schedule arranged with 20 inter-response intervals (IRI) averaging $15 \mathrm{~s}$ was used. The 20 IRIs used in progression were generated logarithmically based on the equation developed by Fleshler and Hoffman (1962). Under this schedule, the first IRI was set at $16.874 \mathrm{~s}$ and was the same for all subjects. This IRI value was specifically chosen because of its close proximity in value to the average interval of VI schedule used in this study. After the first reinforcement, the 20 IRIs were randomized and each subject was exposed to a different IRI progression. An IRI that was in effect at a given moment had to elapse before reinforcement of the first correct response occurred. Moreover, the IRI that was in effect did not progress to the next IRI until the first response on the correct lever was made. Incorrect lever presses had no programmed consequences.

\section{Stimulus generalization tests}

Once subjects met the discrimination criterion of $\geq 80 \%$ responses on the condition-appropriate lever before delivery of the first reinforcer for eight of the ten most recent sessions, stimulus generalization tests began. Test sessions for all subjects regardless of training schedule consisted of 2.5-min extinction trials. The number of responses on each lever was recorded in each of five 30 -s bins over the 2.5 -min tests. The percentage of responses on the drug-appropriate lever was calculated to index stimulus generalization. All subjects were administered stimulus generalization tests with cocaine $(0,1.25,2.5,5.0,10.0 \mathrm{mg} / \mathrm{kg}$, IP), MDMA (0, 0.375, $0.75,1.5 \mathrm{mg} / \mathrm{kg}, \mathrm{IP})$, d-amphetamine $(0,0.5,1.0,2.0 \mathrm{mg} / \mathrm{kg}$, IP), and LSD ( $0,0.04,0.08 \mathrm{mg} / \mathrm{kg}$, IP). These doses are comparable to the doses used in other drug discrimination studies (e.g., Khorana et al. 2004; Oberlender and Nichols 1988, White and Appel 1982). Thus, the use of these doses allows meaningful comparisons to be made between the present results and those of other drug discrimination investigations. Tests were conducted once or twice a week, and each dose was tested independently during a single extinction session. Training sessions were conducted between tests, and subjects were required to meet the $80 \%$ minimum criterion before reinforcer delivery under both training conditions before each test.

\section{Data analysis}

The number of sessions-to-criterion (STC) completed by all four groups was compared using a two-factor ANOVA, with drug and schedule as the two factors. Animals that did not meet criterion were excluded from statistical analyses. Response rates and the number of reinforcers earned by both FR 20 and VI 15-s training groups during drug and vehicle training conditions were compared using a two-way repeated measures ANOVA, with drug and session as the two factors. Response rates were expressed as the number of responses per second.

Results of stimulus generalization tests were plotted in dose-response curves using quantitative measures. For each subject, the percentage of drug-appropriate responses was determined and group means were calculated for each dose. To be included in the group mean, an animal had to make $\geq 5$ responses during the entire test session. Response rates during stimulus generalization tests were also plotted in dose-response functions as group means.

Full substitution for MDMA or cocaine was defined as a group mean of at least $80 \%$ drug-appropriate responses. Partial substitution was defined as drug-appropriate responses between 20 and $80 \%$, and no substitution was defined as $\leq 20 \%$ drug-appropriate responses. Nonlinear regression analysis was used to calculate and analyze effective dose $50\left(\mathrm{ED}_{50}\right)$ values from dose-response curves. A one-way repeated measures ANOVA, with dose as the main factor, was conducted on individual dose-response curves for each individual group. A split-plot analysis was also conducted to compare between-subject factors (schedule assignment) and within subject factors (doses of each test drug). All ANOVA tests that were significant were followed by a post hoc Student-Newman-Keuls (SNK) test for pairwise comparisons. Statistical significance was defined as $p<0.05$ for all tests. 
All figures, confidence intervals, and $\mathrm{ED}_{50} \mathrm{~s}$ were graphed and calculated with GraphPad Prism 4 (GraphPad Software, San Diego, CA). All ANOVAs and post hoc comparisons were calculated with both SigmaStat 3.11 (Systat Software, Point Richmond, CA) and SPSS 13 (SPSS, Chicago, IL).

\section{Results}

Discrimination acquisition

Figure 1 depicts the discrimination acquisition curves for each of the four training groups, COC-FR, COC-VI, MDMA-FR, and MDMA-VI. In these figures, the term "sessions" refers to the $n$th exposure to either drug or saline. Percentages were based on drug-appropriate responses before the first reinforcement under either the FR 20 or the VI 15-s schedule. Both COC-FR and COC-VI groups met the discrimination criterion within $40.4 \pm 6.19$ sessions (range 20-70, $n=8$; Fig. 1a) and $88.4 \pm 18.38$ (range 31-177, $n=8$ ), respectively (Fig. 1b). The MDMA-FR and the MDMA-VI group met the discrimination criterion within $60.5 \pm 7.37$ (range 23-80, $n=8$; Fig. 1c) and 116.6 \pm 13.8 (range 77-161, $n=7$; Fig. 1d) respectively. One subject in the MDMA-VI group failed to meet the criterion and was therefore excluded from statistical analyses.

There was a significant main schedule effect on the number of STC completed $[F(1,27)=17.589, p<0.001]$. SNK compar- isons of FR 20 vs VI 15-s schedules were significant with both MDMA $(p=0.004)$ and cocaine $(p=0.01)$. There was no main drug effect and no drug $\times$ schedule interaction effect on STC completed. Thus, compared to the VI 15-s schedule, the FR 20 schedule facilitates rapid discrimination acquisition by both cocaine and MDMA.

Response rates during acquisition

For response rates and number of reinforcers earned, pairwise comparisons between consecutive drug and vehicle training sessions were conducted at every $25 \pm 1$ session. For example, session 25 was a vehicle training session. It was therefore compared to the closest drug training session, which was session 24. Figure 2 depicts mean $( \pm$ SEM) response rates under saline and drug conditions for each of the four training groups during the same training sessions illustrated in Fig. 1. Significant differences between response rates during cocaine $(\mathrm{C})$ and vehicle $(\mathrm{V})$ conditions were found between sessions $125(\mathrm{~V})$ and126 (C), $149(\mathrm{C})$ and $150(\mathrm{~V}), 174(\mathrm{~V})$ and $175(\mathrm{C})$ (SNK test, all $p$ values $<0.05$ ) for COC-FR rats (Fig. 2a). With the exception of sessions $174(\mathrm{~V})$ and $175(\mathrm{C})$, no significant differences in cocaine and vehicle response rates were found with COC-VI rats (Fig. 2b). As depicted in Fig. 2c, significant differences between MDMA (M) and vehicle (V) response rates were found with MDMA-FR rats between training sessions $75(\mathrm{M})$ and $76(\mathrm{~V}), 99(\mathrm{M})$
Fig. 1 Discrimination acquisition in COC-FR (a), COC-VI (b), MDMA-FR (c), and MDMA-VI groups (d). Points represent the group mean percentage of drug-lever responses before delivery of the first reinforcer during each session a FR 20 and cocaine

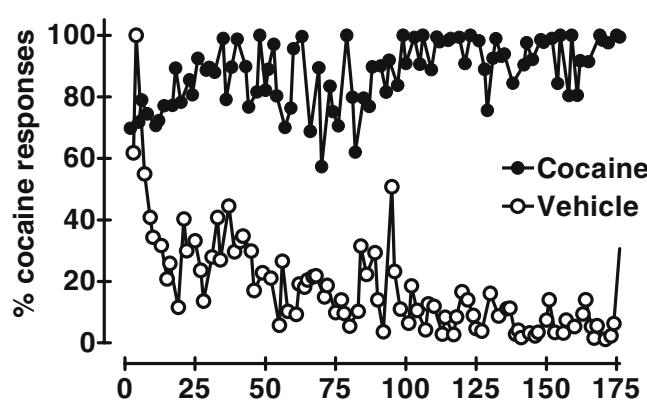

c FR 20 and MDMA

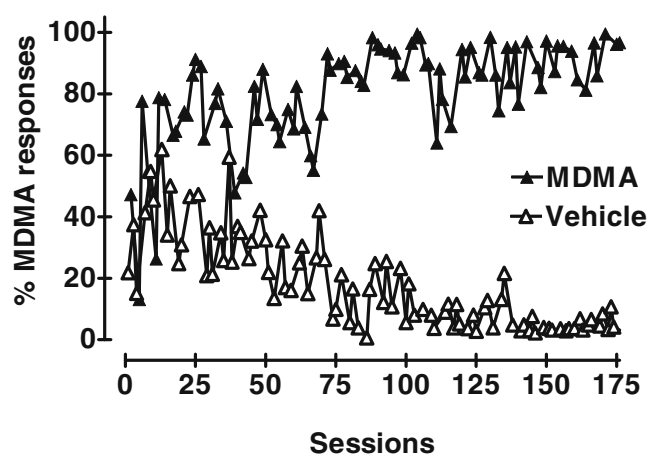

b VI $15 \mathrm{~s}$ and cocaine

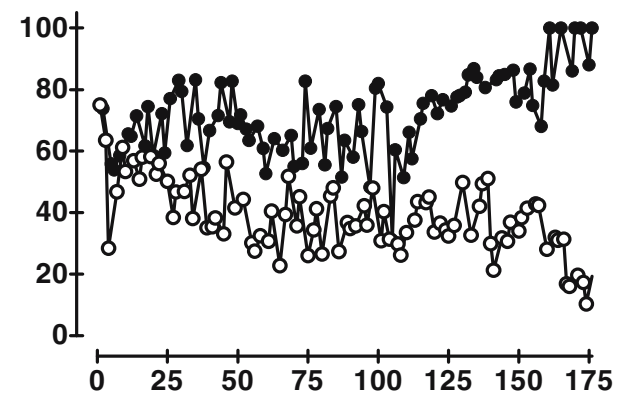

d VI $15 \mathrm{~s}$ and MDMA

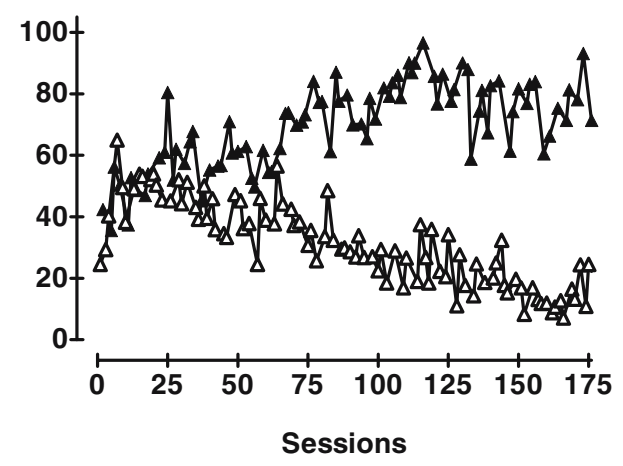


Fig. 2 Mean response rates earned by COC-FR (a), COC-VI (b), MDMA-FR (c), and MDMA-VI groups (d). Points represent the group mean number of reinforcers earned during each session. Verticallines depict standard errors $( \pm$ SEM). For clarity, only every 25 th $( \pm 1)$ session is shown. An asterisk indicates significant differences between drug and vehicle training sessions with respect to response rates $(p<0.05)$ a FR 20 and cocaine
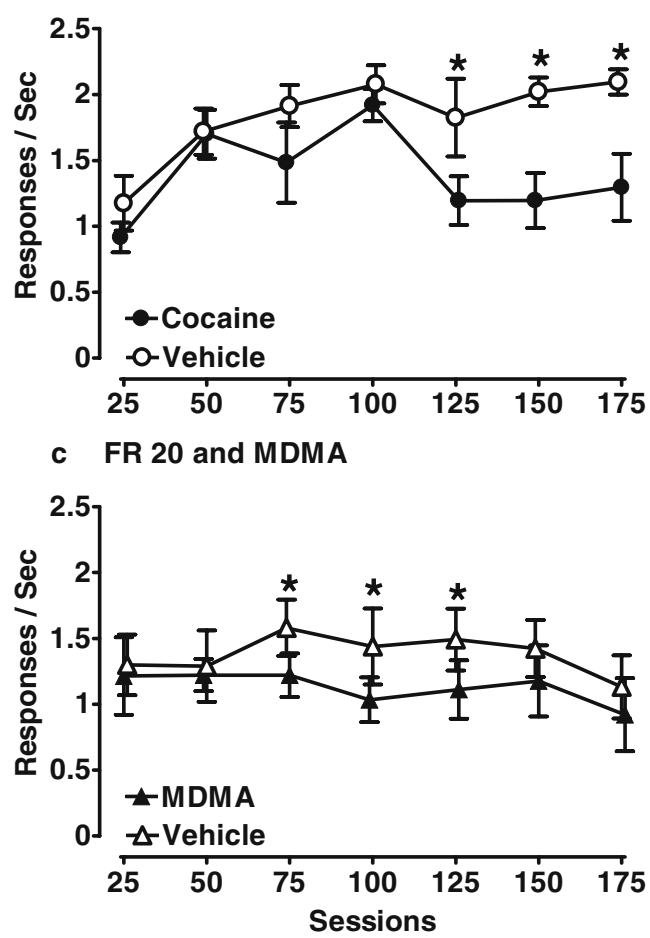

b VI 15-s and cocaine
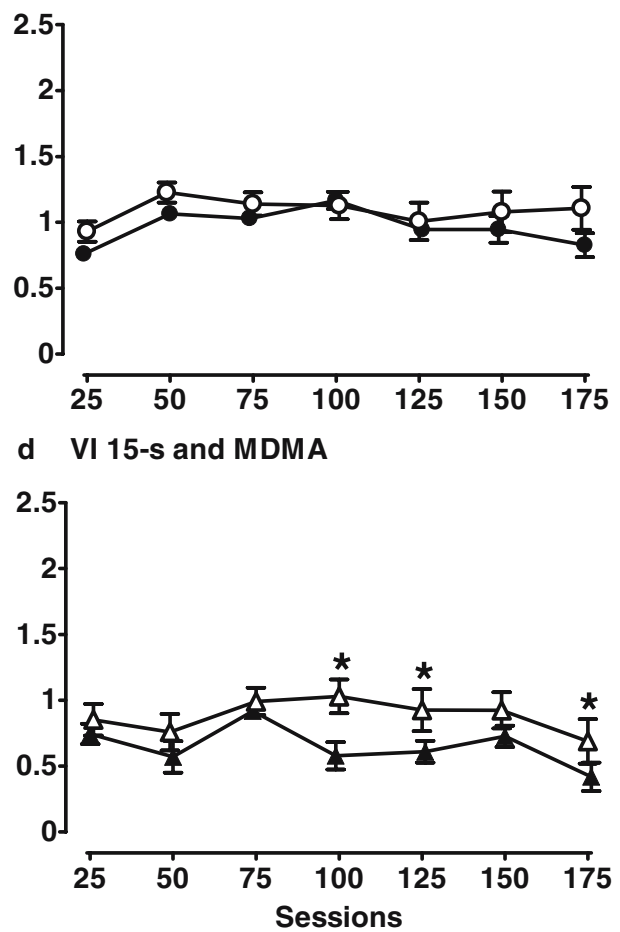

and $100(\mathrm{~V})$, and $125(\mathrm{~V})$ and $126(\mathrm{M})$ (SNK test, all $p$ values $<0.05)$. Significant differences were also found between MDMA and vehicle responses with MDMA-VI rats between training sessions $99(\mathrm{M})$ and $100(\mathrm{~V}), 125(\mathrm{~V})$ and $126(\mathrm{M})$, and $175(\mathrm{~V})$ and $176(\mathrm{M})$ (SNK test, all $p$ values $<0.05$; Fig. 2d). Thus, differences in response rates appeared later in drug discrimination training with all groups except COC-VI rats.

Number of reinforcers earned during acquisition

Figure 3 depicts mean $( \pm$ SEM) number of reinforcers earned for each of the four training groups under drug and vehicle conditions during the same training sessions illustrated in Fig. 1. These data were analyzed in a similar manner as response rates, described above. Significant differences between cocaine and vehicle conditions were found with respect to the number of reinforcers earned by COC-FR rats between sessions 149 (C) and 150 (V), and 174 (V) and 175 (C) (SNK test, all $p$ values $<0.05$; Fig. 1a). No significant differences between cocaine and vehicle conditions were found with respect to the number of reinforcers earned by COC-VI (Fig. 3b). As depicted in Fig. 3c, significant differences between MDMA and vehicle conditions were found with respect to the number of reinforcers earned by MDMA-FR rats between sessions 74 $(\mathrm{M})$ and $75(\mathrm{~V}), 99(\mathrm{M})$ and $100(\mathrm{~V})$, and between sessions 125 (V) and $126(\mathrm{M})$ (SNK test, all $p$ values $<0.05$ ). No significant differences between MDMA and vehicle con- ditions were found with respect to the number of reinforcers earned by MDMA-VI rats (Fig. 3d). Thus, differences in the number of reinforcers earned between drug and vehicle conditions occurred under the FR 20 training schedule but not under the VI 15-s training schedule.

Stimulus generalization tests

Figures 4, 5, 6, and 7 depict the results of stimulus generalization tests with cocaine, MDMA, d-amphetamine, and LSD in that order. As shown in Fig. 4a, cocaine significantly increased the percentage of cocaine-appropriate responses in a dose-dependent manner in both COC-FR $[F(4,28)=12.56, p<0.001]$ and COC-VI $[F(4,27)=13.6$, $p<0.001]$ groups. SNK comparisons of saline vs different doses of cocaine were significant, but only at $5-10 \mathrm{mg} / \mathrm{kg}$ with COC-FR rats (all $p$ values $<0.05$ ) and $2.5-10 \mathrm{mg} / \mathrm{kg}$ with COC-VI rats (all $p$ values $<0.05$ ). Cocaine $\mathrm{ED}_{50}$ values were $2.22 \mathrm{mg} / \mathrm{kg}$ (95\% confidence intervals, $1.82-$ $2.62 \mathrm{mg} / \mathrm{kg}$ ) for COC-FR and $1.25 \mathrm{mg} / \mathrm{kg}$ (95\% CI, 0.96$1.53 \mathrm{mg} / \mathrm{kg}$ ) for COC-VI groups. Split-plot analysis of cocaine substitution tests revealed no significant differences between COC-FR and COC-VI groups with respect to the percentage of cocaine-appropriate responses. As shown in Fig. 4b, cocaine only engendered 54.52 $\pm 16.29 \%$ MDMAappropriate responses in MDMA-FR rats and $58.48 \pm 9.480 \%$ MDMA-appropriate responses in MDMA-VI rats. Nevertheless, a significant main dose effect was found with MDMA-VI rats $[F(4,28)=12.56, p<0.001]$ but not 
Fig. 3 Mean number of reinforcers earned during training sessions in COC-FR (a), COCVI (b), MDMA-FR (c), and MDMA-VI groups (d). Points represent the group mean number of responses per second within each session. Verticallines depict standard errors ( \pm SEM). For clarity, only every 25 th $( \pm 1)$ session is shown. An asterisk indicates significant differences between drug and vehicle training sessions with respect to number of reinforcers earned $(p<0.05)$

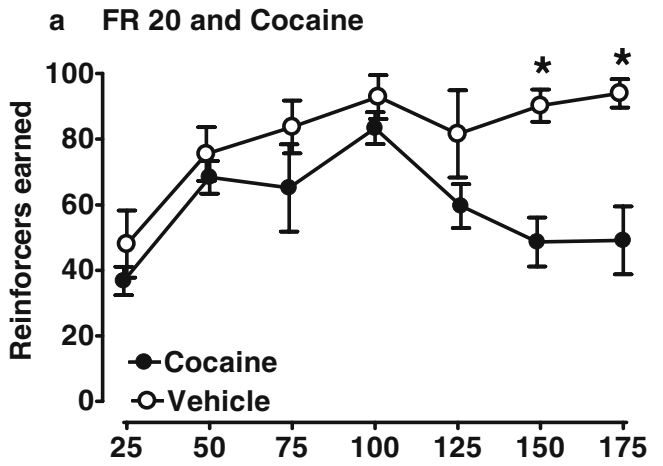

c FR 20 and MDMA

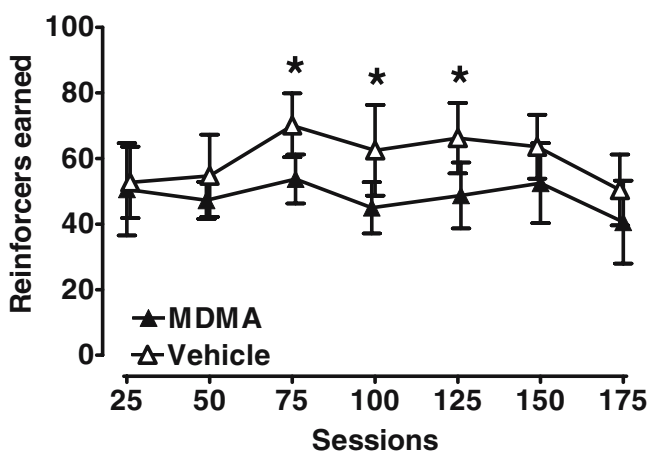

b VI 15-s and Cocaine

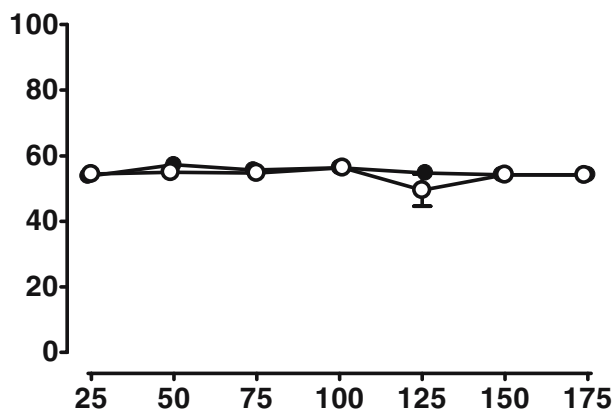

d VI 15-s and MDMA

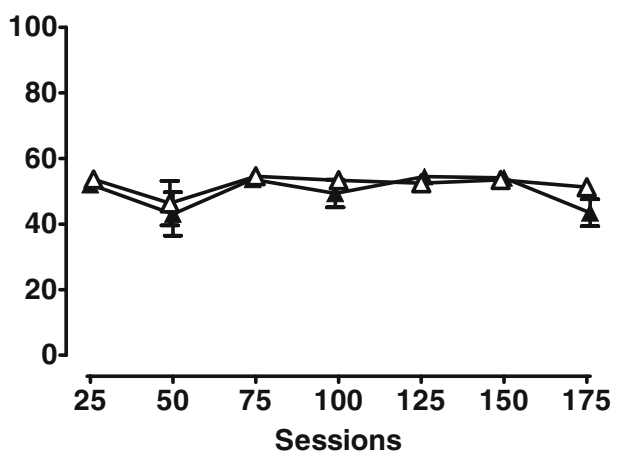

MDMA-FR rats. No significant differences between MDMA-FR and MDMA-VI groups were found with respect to the percentage of MDMA-appropriate responses. Thus, cocaine failed to substitute for MDMA in all MDMA-trained animals. As depicted in Fig. 4c,d, overall response rates were significantly lower in COC-VI rats compared to COC-FR rats $[F(1,12)=19.85, p=0.001]$, but were similar in both MDMA-FR and MDMA-VI rats. Response rates remained constant in cocaine-trained rats (Fig. 4c), but were significantly reduced in MDMA-trained rats $[F(4,4)=5.24, p=0.05$; Fig. $4 \mathrm{~d}]$.
Fig. 4 Cocaine dose-response functions in rats trained to discriminate cocaine $(\mathbf{a}, \mathbf{c})$ or MDMA (b, d) under an FR 20 or a VI 15-s reinforcement schedule. Points in graphs a and b represent the mean $( \pm \mathrm{SEM})$ percentage of drug-appropriate responses during 2.5 -min test sessions. Points in graphs $\mathbf{c}$ and d depict the mean $( \pm$ SEM $)$ number of responses per second

\section{a Cocaine tests with cocaine-trained rats}
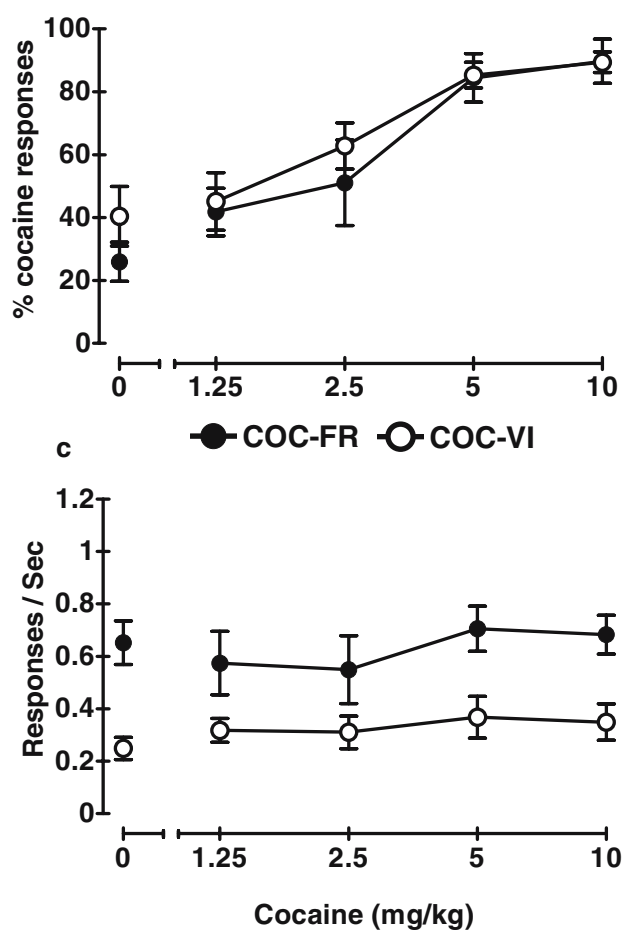

b Cocaine tests with MDMA-trained rats
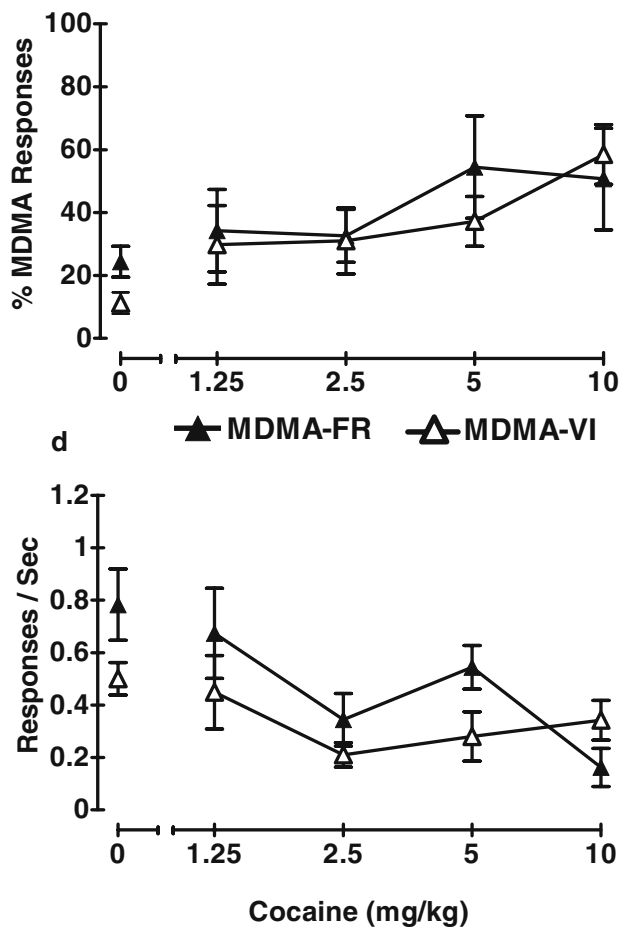
Fig. 5 MDMA dose-response functions in rats trained to discriminate cocaine $(\mathbf{a}, \mathbf{c})$ or MDMA (b, d) under an FR 20 or a VI 15-s reinforcement schedule. Points in the graphs $\mathbf{a}$ and $\mathbf{b}$ represent the mean $( \pm$ SEM) percentage of drugappropriate responses during 2.5-min test sessions. Points in graphs $\mathbf{c}$ and $\mathbf{d}$ depict the mean $( \pm$ SEM) number of responses per second. The symbol $\uparrow$ indicates that more than half of the test subjects were disrupted at that specific dose

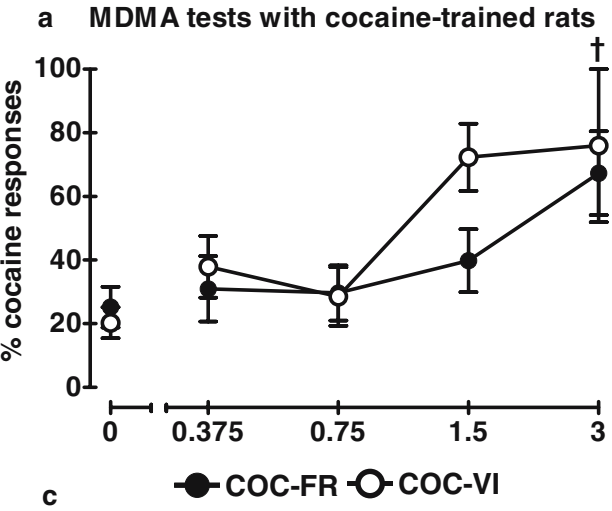

b MDMA tests with MDMA-trained rats

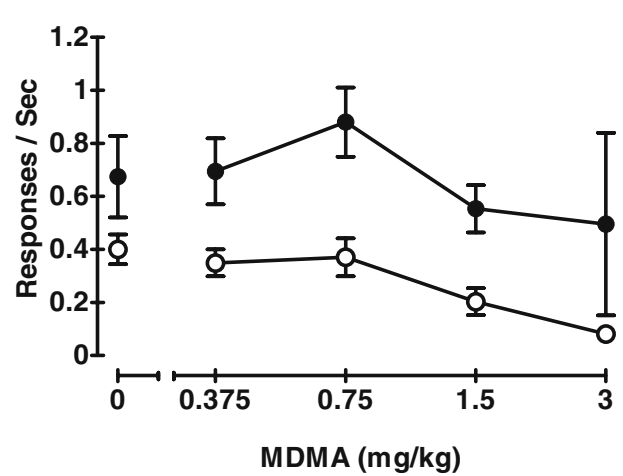

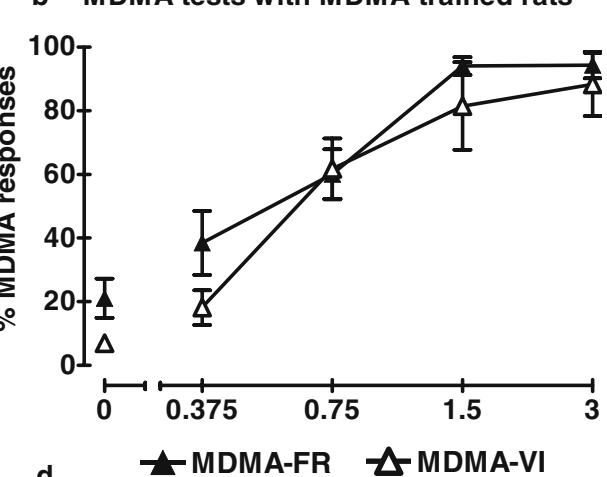

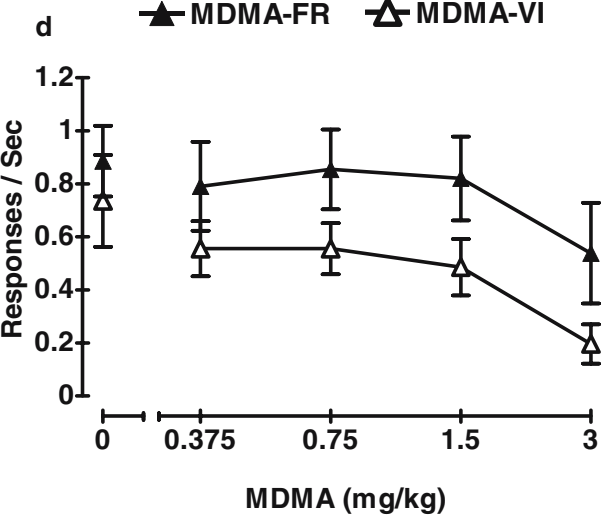

As shown in Fig. 5a, MDMA failed to substitute completely for cocaine. Nevertheless, MDMA significantly increased the percentage of cocaine-appropriate responses in a dose-dependent manner with COC-FR $[F(4,23)=3.45$, $p=0.023]$ and COC-VI rats $[F(4,23)=7.06, p<0.001$; Fig. 5a]. Significant differences between MDMA and saline were found but only at $3 \mathrm{mg} / \mathrm{kg}$ with COC-FR rats (SNK test, $p=0.0015$ ) and $1.5-3 \mathrm{mg} / \mathrm{kg}$ with COC-VI rats (SNK test, all $p$ values $<0.05$ ). No significant differences between COC-FR and COC-VI groups were found with respect to the percentage of cocaine-appropriate responses after MDMA administration. MDMA also increased the percentage of MDMA-appropriate responses with both MDMA-FR [ $F$ $(4,24)=23.11, p<0.001]$ and MDMA-VI rats $[F(4,19)=16.58$,
Fig. 6 Results of stimulus generalization tests with d-amphetamine in rats trained to discriminate cocaine $(\mathbf{a}, \mathbf{c})$ or MDMA (b, d) under an FR 20 or a VI 15-s reinforcement schedule. Points in graphs a and b represent the mean $( \pm \mathrm{SEM})$ percentage of drug-appropriate responses during 2.5 -min test sessions. Points in graphs $\mathbf{c}$ and d depict the mean $( \pm$ SEM) number of responses per second a AMPH tests with cocaine-trained rats
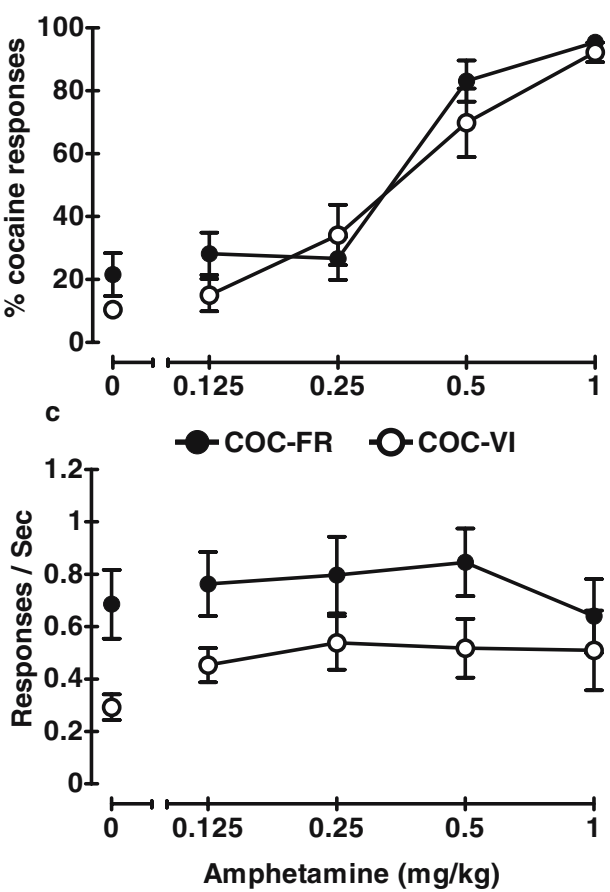

b AMPH tests with MDMA-trained rats
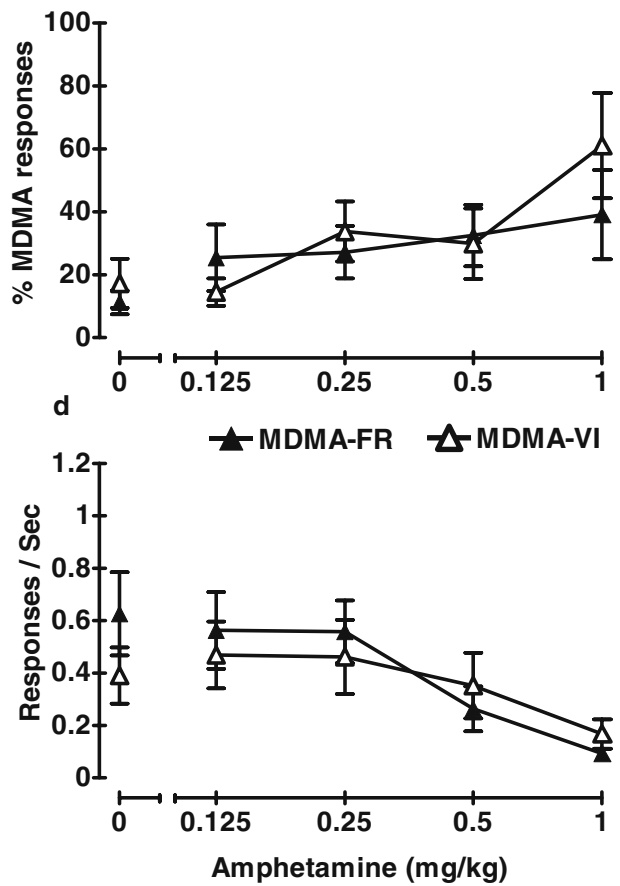
Fig. 7 Results of LSD stimulus generalization tests in rats trained to discriminate cocaine $(\mathbf{a}, \mathbf{c})$ or MDMA $(\mathbf{b}, \mathbf{d})$ under an FR 20 or a VI 15-s reinforcement schedule. Points in graphs $\mathbf{a}$ and $\mathbf{b}$ represent the mean ( \pm SEM) percentage of drugappropriate responses during 2.5-min test sessions. Points in graphs $\mathbf{c}$ and $\mathbf{d}$ depict the mean $( \pm$ SEM) number of responses per second. The symbol $\uparrow$ indicates that more than half of the test subjects were disrupted at that specific dose a LSD tests with cocaine-trained rats

b LSD tests with MDMA-trained rats

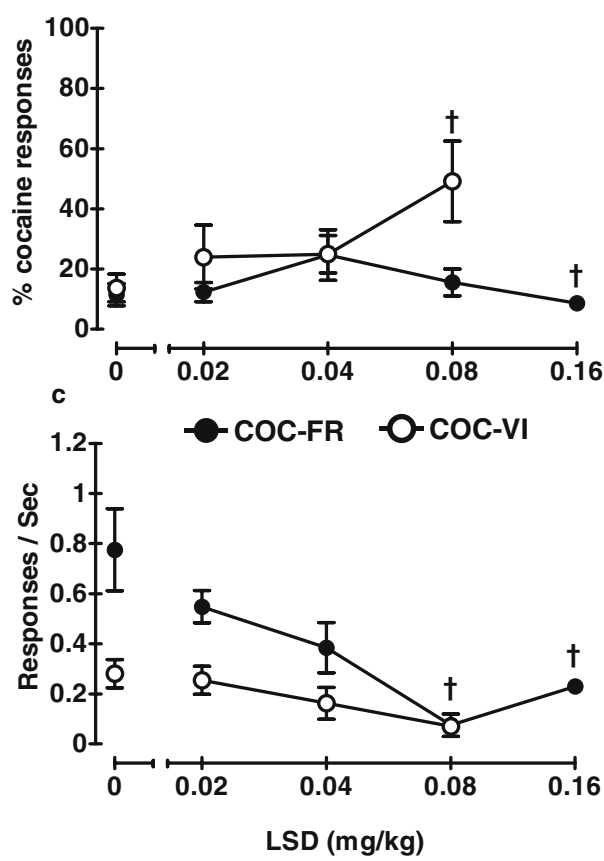

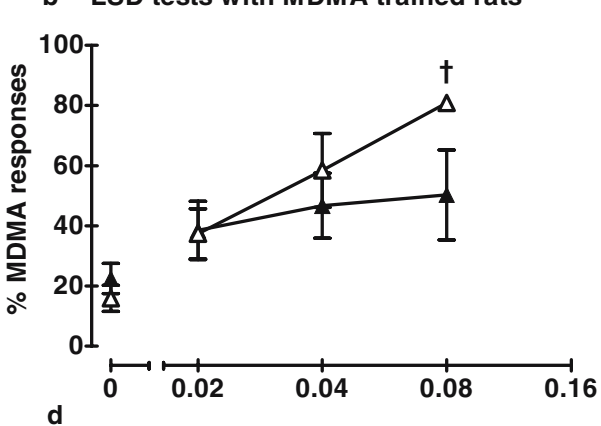

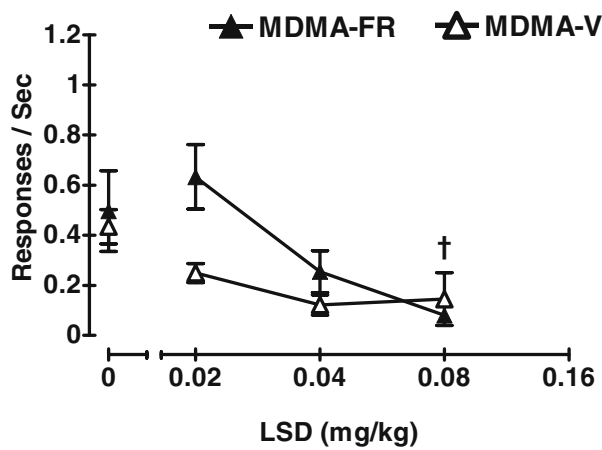

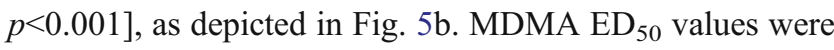
$0.61 \mathrm{mg} / \mathrm{kg}(95 \% \mathrm{CI}, 0.46-0.75 \mathrm{mg} / \mathrm{kg})$ for MDMA-FR rats and $0.81 \mathrm{mg} / \mathrm{kg}(95 \% \mathrm{CI}, 0.59-1.03 \mathrm{mg} / \mathrm{kg})$ for MDMA-VI rats. As depicted in Fig. 5c,d, overall response rates were significantly lower in COC-VI rats compared to COC-FR rats $[F(1,13)=2.05, p=0.007]$ and in MDMA-VI rats compared to MDMA-FR rats $[F(1,7)=7.81, p=0.027]$. There was a significant dose-dependent decrease in response rates in cocaine-trained rats $[F(3,3)=3.75, p=0.018$; Fig. 4c] and in MDMA-trained rats $[F(4,4)=4.35, p=0.007$; Fig. 4d].

As expected, d-amphetamine substituted fully for cocaine in a dose-dependent manner (Fig. 6a) with both COC-FR $[F(4,28)=42.52, p<0.001]$ and COC-VI $[F(4,28)=$ $30.05, p<0.001]$ rats. Significant differences between $\mathrm{d}-$ amphetamine and saline were found but only at $0.5-1 \mathrm{mg} / \mathrm{kg}$ with COC-FR rats (SNK test, all $p$ values $<0.05)$ and 0.25 $1 \mathrm{mg} / \mathrm{kg}$ with COC-VI rats (SNK test, all $p$ values $<0.05$ ). No significant differences in the percentage of cocaineappropriate responses were found between COC-FR and COC-VI groups after d-amphetamine administration. The d-amphetamine $\mathrm{ED}_{50}$ was lower in the COC-FR group $(0.36 \mathrm{mg} / \mathrm{kg} ; 95 \% \mathrm{CI}, 0.22-0.51 \mathrm{mg} / \mathrm{kg})$ compared to the COC-VI group $(0.48 \mathrm{mg} / \mathrm{kg} ; 95 \%$ CI, 0.32-0.64 mg/kg). As depicted in Fig. 6b, d-amphetamine failed substitute for MDMA in both MDMA-FR and MDMA-VI animals. As depicted in Fig. 6c,d, response rates were significantly lower in COC-VI rats compared to COC-FR rats $[F(1,14)=$ 5.75, $p=0.031]$ and in MDMA-VI rats compared to MDMA-FR rats $[F(1,5)=23.93, p=0.05]$. Nevertheless, response rates remained constant in cocaine-trained rats but were significantly reduced in MDMA-trained rats $[F(4,4)=$ 5.29, $p=0.004]$.

LSD failed to engender more than 50\% cocaineappropriate responses in both COC-FR or COC-VI groups (Fig. 7a). No significant differences between COC-FR and COC-VI were found. As shown in Fig. 7b, LSD produced only 50\% MDMA-appropriate responses in the MDMA-FR rats, but produced complete substitution in the MDMA-VI group $[F(2,11)=8.82, p=0.005]$. There was a significant dose-dependent increase in the percentage of MDMA responses at $0.04 \mathrm{mg} / \mathrm{kg}$ of LSD (SNK test, $p=0.004$ ). $\mathrm{ED}_{50}$ for LSD in MDMA-VI rats is $0.034 \mathrm{mg} / \mathrm{kg}(95 \% \mathrm{CI}$, $0.022-0.054 \mathrm{mg} / \mathrm{kg}$ ). As depicted in Fig. 7c,d, response rates were significantly lower in COC-VI rats compared to COC-FR rats $[F(1,9)=6.49, p=0.031]$, but were similar in both MDMA-FR and MDMA-VI rats. Response rates remained constant in cocaine-trained rats but were significantly reduced in MDMA-trained rats $[F(2,2)=11.74$, $p<0.001]$.

\section{Discussion}

The present study compared two reinforcement schedules commonly implemented in drug discrimination investigations to assess the extent to which reinforcement schedules modulate the development of discriminative stimulus control by either MDMA or cocaine and to determine the effects of differential training histories on stimulus gener- 
alization between these two substances. The results appear to be consistent with previous conclusions by other investigators that reinforcement schedules influence the development of discriminative stimulus control by psychoactive drugs (Overton 1979; DeVry et al. 1984; Stolerman 1989a,b; Snodgrass and McMillan 1991; Craft et al. 1998; McMillan and Wenger 1984; McMillan et al. 2001). Results depicted in the discrimination acquisition curves (Fig. 1) and statistical comparisons of the number of sessions to criterion indicate that the acquisition of cocaine and MDMA discrimination was facilitated much more rapidly under the FR 20 schedule than under the VI 15-s schedule. McMillan et al. (2001) explained that such differences may be due to the resetting component that was present in the FR schedule. That is, the FR resetting component automatically resets the response counter when an incorrect lever press was made, which delayed reinforcement delivery. Thus, under the FR 20 schedule, there is greater consequential control compared to the VI 15-s schedule because incorrect responses are punished under the FR 20 schedule, but not under the VI 15-s schedule. This punishment feature of the resetting FR schedule may account for the better drug discrimination acquisition. Future investigations regarding the influence of punishment contingencies on the acquisition of drug discrimination are warranted. This could be examined by adding consequences for incorrect responses under the VI schedule (e.g., time-out after incorrect responses).

Alternatively, more rapid discrimination acquisition under the FR 20 schedule might be attributed to differences in response rates and/or differences in the frequency of reinforcement during drug and vehicle training conditions under the FR 20 schedule, as such differences were not observed under the VI 15-s schedule. Future investigations may address the role of reinforcement frequency by setting a limit on the maximum number of reinforcers that can be earned under the FR 20 schedule, so that the number of reinforcers earned under drug conditions and under saline conditions are equivalent. A second possible solution would be to yoke the number of reinforcements earned by animals trained under the VI schedule to the number of reinforcements generated from animals trained under the FR 20 schedule (Ferster and Skinner 1957). This yoked box system would control for both rate of reinforcements and response rates of animals trained under either FR or VI schedules. With such procedures, differences between drug and saline conditions with respect to response rates and number of reinforcers earned may not be as prominent as was the case in the present study.

Despite differences in discrimination acquisition between FR 20-trained subjects and VI 15-s trained subjects, only moderate differences were observed in the results of stimulus generalization tests. Although the $\mathrm{ED}_{50}$ for cocaine was lower in the COC-VI group compared to the COC-FR group, this difference was likely due to higher drug-appropriate responding after the $0 \mathrm{mg} / \mathrm{kg}$ test dose and, thus, a flatter dose response function in the COC-VI group. The greatest difference between the COC-VI and COC-FR groups was observed after $1.5 \mathrm{mg} / \mathrm{kg}$ MDMA, which engendered greater cocaine-lever responding in the COC-VI group (72\%) than in the COC-FR group $(40 \%)$. Nevertheless, MDMA did not fully substitute for cocaine in either training group. Dose-response functions were similar in the MDMA-VI and MDMA-FR groups, and both cocaine and d-amphetamine produced only partial substitution for MDMA in both groups. The present results are inconsistent with the results of Khorana et al. (2004), who reported that $10 \mathrm{mg} / \mathrm{kg}$ of cocaine engendered 75\% MDMA-appropriate responses, whereas $11 \mathrm{mg} / \mathrm{kg}$ of cocaine engendered $88 \%$ MDMA-appropriate responses and therefore substituted for MDMA. However, the present results are consistent with the results by Schechter (1998), who reported that cocaine does not substitute for MDMA. Moreover, the possibility that the discrepant results between these two previous studies are related to the use of different reinforcement schedules is unlikely, given the results of the current study.

There was some evidence for differential effects of LSD in FR 20 trained animals and VI 15-s trained animals. In rats trained to discriminate MDMA, $0.08 \mathrm{mg} / \mathrm{kg}$ LSD produced only partial substitution in the MDMA-FR 20 group $(50 \%)$, while this dose produced full substitution in the MDMA-VI 15-s group (81\%). Even in rats trained to discriminate cocaine, this dose of LSD produced greater drug-appropriate responding in the VI 15-s training group (49\%) compared to the FR 20 training group $(16 \%)$. These data must be considered tentatively, however, because this dose of LSD severely reduced response rates such that only two to three animals completed the response requirement and were included in graphical and statistical analyses.

In summary, results of the present investigations demonstrated that an FR 20 reinforcement schedule established discriminative stimulus control by MDMA and by cocaine more rapidly compared to a VI 15-s schedule. Results from the present study are consistent with results from previous studies that showed different reinforcement schedules modulate the discriminative stimulus effects of psychoactive drugs and extend these findings to MDMA and cocaine. Other variables such as differences in response rates and/or reinforcement frequency generated by these schedules may play a role in the development of stimulus control. However, further investigations targeted at controlling these variables are required to evaluate the extent to which they may modulate the discriminative stimulus properties of these drugs. Finally, despite differences in discrimination acquisition under FR 20 and VI 15-s reinforcement schedules, results of stimulus generalization 
varied only minimally in animals with differential training histories. Regardless of schedule, MDMA and cocaine failed to produce cross-generalization in this study.

Acknowledgements The authors acknowledge the exceptional technical assistance provided by Bismarck J. Manes and William A. Lizik.

\section{References}

Anderson KG, Van Haaren F (1999) Cocaine discrimination and timecourse effects in male and female Wistar rats. Eur J Pharmacol 382:69-74

Anderson KG, Van Haaren F (2000) Effects of SCH-23390 and raclopride on cocaine discrimination in male and female Wistar rats. Pharmacol Biochem Behav 65:671-675

Baker LE, Broadbent J, Michael EK, Matthews PK, Metosh CA, Saunders RB, West WB, Appel JB (1995) Assessment of the discriminative stimulus effects of the optical isomers of ecstasy (3,4-methylenedioxymethamphetamine; MDMA). Behav Pharmacol 6:263-275

Baker LE, Pynnonen D, Poling A (2004) Influence of reinforcer type and route of administration on gamma-hydroxybutyrate discrimination in rats. Psychopharmacology (Berl) 174:220-227

Bondavera T, Wesolowska A, Dukat M, Lee M, Young R, Glennon RA (2005) S(+)-and R(-)N-Methyl-1-(3,4-methylenedioxyphenyl)-2-aminopropane (MDMA) as discriminative stimuli: effect of cocaine. Pharmacol Biochem Behav 531-538

Brauer LH, Goudie AJ, de Wit H (1997) Dopamine ligands and the stimulus effects of amphetamine: animal models versus human laboratory data. Psychopharmacology (Berl) 130:2-13

Broadbent J, Michael EK, Appel JB (1989) Generalization of cocaine to the different isomers of 3,4-methylenedioxyamphetamine and 3,4-methylenedioxymethamphetamine: effects of training dose. Drug Dev Res 16:443-450

Colpaert FC, Janssen PAJ (1981) Factors regulating drug cue sensitivity: the effect of frustrative non-reward in fentanyl-saline discrimination. Arch Int Pharmacodyn Ther 254:241-251

Craft RM, Stratmann JA (1996) Discriminative stimulus effects of cocaine in female versus male rats. Drug Alcohol Depend 42: $27-37$

Craft RM, Kalivas PW, Stratmann JA (1996) Sex differences in discriminative stimulus effects of morphine in the rat. Behav Pharmacol 7:764-778

Craft RM, Morgan CK, Bernal SA (1998) Reinforcement frequency, but not gender, determines sensitivity to discriminative stimulus effects of morphine. Behav Pharmacol 9:357-362

DeVry J, Koek W, Slangen JL (1984) Effects of drug-induced differences in reinforcement frequency on discriminative stimulus properties of fentanyl. Psychopharmacology (Berl) 83:257-261

Extance K, Goudie AJ (1981) Inter-animal olfactory cues in operant drug discrimination procedures in rats. Psychopharmacology (Berl) 73:363-371

Ferster CB, Skinner BF (1957) Schedules of reinforcement. Copley, MA
Fleshler M, Hoffman HS (1962) A progression for generating variable-interval schedules. J Exp Anal Behav 5:529-530

Glennon RA, Rosecrans JA, Young R (1983) Drug-induced discrimination: a description of the paradigm and a review of its specific application to the study of hallucinogenic agents. Med Res Rev 3:289-340

Goudie AJ, Leathley MJ (1993) Drug-discrimination assays. Behavioral Neuroscience: a practical approach. Oxford University Press, New York

Khorana N, Pullagurla MR, Young R, Glennon RA (2004) Comparison of the discriminative stimulus effects of 3,4-methylenedioxymethamphetamine (MDMA) and cocaine: asymmetric generalization. Drug Alcohol Depend 74:281-287

Koek W, Slangen JL (1982) Effects of reinforcement differences between drug and saline sessions on discriminative stimulus properties of fentanyl. In: Colpaert FC, Slangen (eds) Drug discrimination: applications in CNS pharmacology. Elsevier, Amsterdam, pp 343-354

McMillan DE, Wenger GR (1984) Bias of phencyclidine discrimination by the schedule of reinforcement. J Exp Anal Behav 42:51-66

McMillan DE, Hardwick WC, Li M (2001) Discrimination of pentobarbital doses and drug mixtures under fixed-ratio and fixed-interval reinforcement schedules. Behav Pharmacol 12:195-208

Meehan SM, Gordon TL, Schechter MD (1995) MDMA (ecstasy) substitutes for the ethanol discriminative cue in HAD but not LAD rats. Alcohol 12:569-572

National Research Council (1996) Guide for the care and use of laboratory animals. National Academy Press, Washington, DC

Oberlender RA, Nichols DE (1988) Drug discrimination studies with MDMA and amphetamine. Psychopharmacology (Berl) 95:71-76

Overton DA (1979) Influence of shaping procedure and schedules on performance in the two-bar drug discrimination task: a methodological report. Psychopharmacology (Berl) 65:291-298

Schechter MD (1997) Serotonergic mediation of fenfluramine discriminative stimuli in fawn-hooded rats. Life Sci 60(6):PL 83-90

Schechter MD (1998) MDMA-like stimulus effects of hallucinogens in male fawn-hooded rats. Pharmacol Biochem Behav 59:265270

Snodgrass S, McMillan D (1991) Effects of schedule of reinforcement on a pentobarbital discrimination in rats. J Exp Anal Behav $56: 313-329$

Stolerman IP (1989a) Comparison of fixed-ratio and tandem schedules of reinforcement in discrimination of nicotine in rats. Drug Dev Res 16:101-109

Stolerman IP (1989b) Discriminative stimulus effects of nicotine in rats trained under different schedules of reinforcement. Psychopharmacology (Berl) 97:131-138

Stolerman IP, D'Mello G (1981) Role of training conditions in discrimination of central nervous system stimulants. Psychopharmacology (Berl) 73:295-302

White FJ, Appel JB (1982) Training dose as a factor in LSD-saline discrimination. Psychopharmacology (Berl) 76:20-25 